\title{
La evaluación de las aplicaciones de lectura web: un paso más en el proceso de editorialización de la web'
}

\author{
Web Reading Apps' assessment; \\ a step further in the web editorializing process
}

\author{
NOELIA PATÓN RODRÍGUEZ \\ RAQUEL GÓMEZ-DIAZ \\ Universidad de Salamanca \\ España \\ npatonrguez@gmail.com \\ rgomez@usal.es
}

(Recibido:I9-IO-2OI5;

aceptado: 09-02-20I6)

\begin{abstract}
Resumen. En el desarrollo de la lectura digital confluyen una serie de factores que pasan, entre otras cuestiones, por la evolución tecnológica de los dispositivos y el desarrollo de numerosas aplicaciones que facilitan la lectura, al tiempo que, está, provocando cambios en los hábitos de lectura tal y como lo demuestran los diferentes informes. Uno de los desarrollos tecnológicos está relacionado con el diseño de aplicaciones específicas para la lectura en la pantalla del ordenador, son las llamadas aplicaciones de lectura web. En este artículo, tras definir qué son las aplicaciones de lectura web se explican los objetivos y el proceso seguido para la selección, definición y sistematización de los parámetros que servirán para la evaluación de las aplicaciones de lectura web.
\end{abstract}

Palabras clave: aplicaciones de lectura; lectura web; web apps; lectura digital.
Abstract. In the development of the digital reading a series of factors come arise, among them some questions such as the technological evolution of some devices or the development of numerous applications that make reading easier at the same time that they produce changes on reading habits, as it is shown on the different reports. One of this technological developments is related to the design of applications specifically made to read upon the computer's screen, they are called web reading applications. In this article, we define what reading web applications are, as well as the objectives and the process for the selection, definition and systematization of the parameters used to evaluate reading web applications.

Keywords: reading applications; web reading; web apps; digital reading.

\footnotetext{
I Para citar este artículo: Patón Rodríguez, Noelia y Gómez-Díaz, Raquel (20I6). La evaluación de las aplicaciones de lectura web: un paso más en el proceso de editorialización de la web. Alabe 14. [www.revistaalabe.com]

DOI: IO.I5645/Alabe20i6.I 4.5
} 


\section{Introducción}

Se entiende por lectura digital aquella que se hace sobre un soporte informático (Alonso-Arévalo, Gómez-Díaz, \& Cordón-García, 20I5) e implica la capacidad de un individuo para comprender, reflexionar, utilizar y compartir los contenidos de los textos digitales, permitiendo el acceso a la información, al conocimiento, al arte y la cultura (Cordón-García, Alonso-Arevalo, Gómez-Díaz, \& Linder, 2OI3). Se puede practicar con diversos dispositivos (ordenador, e-reader, tablet o smartphone) que son los que facilitan la decodificación del mensaje. Tal y como muestran las distintas encuestas que tratan el tema de la lectura digital (Conecta, 2OI4), (Zickuhr \& Rainie, 2OI4), ("Encuesta de hábitos y prácticas culturales en España: Septiembre de 20I5," 20I5) es una práctica que va en aumento.

A diferencia de la lectura que se hace en los soportes analógicos, la lectura digital es más fragmentaria, menos lineal, menos profunda, pero más extensiva, y en ella son de gran importancia las derivaciones multimedia (Cordón-García, J. A., \& Jarvio-Fernández, A. O., 20I5) y además transcurre entre distintos dispositivos (e-reader, tablet, smartphone y ordenador). Para que se haya producido el cambio a la lectura digital ha sido necesaria la confluencia de una serie de factores: la popularidad de conexión wifi que permite estar permanente conectados; el desarrollo de la Web 2.o que aumentó considerablemente el número de contenidos web; la evolución tecnológica que ha permitido el desarrollo de dispositivos móviles a precios cada vez más asequibles, primero los e-reader y las tabletas después, los cuales impulsaron la lectura digital; las numerosas aplicaciones para la lectura electrónica, algunas con la limitación de su adaptación solo a determinados formatos, y por tanto solo útiles para algunos contenidos; y finalmente el desarrollo de aplicaciones de lectura web, que permiten al usuario realizar una lectura más cómoda y sencilla de todo lo que encuentra navegando por la web e integran algunas de las funcionalidades de las aplicaciones de lectura desarrolladas para las tablets.

Actualmente existen diversos estudios sobre aplicaciones de libros electrónicos y sus dispositivos, los cuales evalúan, comparan y analizan las diferentes herramientas y prestaciones que poseen cada uno de ellos. Sin embargo, las aplicaciones de lectura web son herramientas emergentes, de las cuales aún no se han realizado estudios sobre ellas, ni tampoco comparaciones entre las distintas aplicaciones existentes. Por ello, el objetivo principal de este artículo es evaluar y comparar dichas aplicaciones para deducir si todas ellas presentan las mismas funcionalidades o por el contrario, existen diferencias entre ellas. Esto conlleva crear y establecer unos parámetros e indicadores específicos que permitan evaluar este tipo de aplicaciones. Hasta ahora, existían estudios sobre la medición de páginas web con parámetros e indicadores específicos que medían la legibilidad y calidad de los sitios web. Sin embargo, estas herramientas no son útiles para evaluar las aplicaciones de lectura web, ya que no poseen las mismas prestaciones. 
Este artículo se centra en las aplicaciones específicas para leer en la pantalla del ordenador aportando una serie de parámetros que servirán para la evaluación de las citadas aplicaciones.

\section{Las aplicaciones de lectura para la web}

Las aplicaciones de lectura la web o web app son aquellas aplicaciones que funcionan dentro del navegador de cualquier dispositivo independientemente de qué sistema operativo utilice. Hay que distinguirlas de las aplicaciones nativas que han sido diseñadas para utilizarse en una plataforma concreta y se pueden descargar desde los Market Places de los distintos sistemas operativos. Según Henning (20I4) las aplicaciones nativas suelen ser las más utilizadas por los usuarios debido a que facilitan un acceso rápido y fácil, ya que las web apps no son fáciles de encontrar, necesitas saber de su existencia y el enlace directo a su página. Aunque no siempre las aplicaciones nativas suelen tener desarrollo web.

Se pueden definir como un servicio que permite al usuario almacenar contenido de la web, facilitando la tarea de trasladar la información que se obtiene navegando por Internet. Permiten gestionar, organizar y guardar de forma sencilla todas las páginas web, noticias o artículos que se consideren importantes para poder consultarlos más tarde. Además incorporan algunas de las características de las aplicaciones de lectura: compartir nuestras impresiones sobre lo que estamos leyendo, cambiar los tipos y el tamaño de la letra... (Gómez-Díaz, R., 20I2, p.29I).

Este tipo de aplicaciones permiten realizar distintas acciones como agrupar los artículos mediante carpetas o etiquetas, así puedes organizar la información atendiendo a la temática y sea más fácil su búsqueda. También se puede compartir dicha información en las redes sociales, ya que estas aplicaciones dan esa opción, también mediante el correo electrónico. Facilitan la lectura de diversa información haciendo que esta sea cómoda y sencilla, permitiendo modificar el aspecto visual del texto al gusto del usuario. Por ejemplo se puede eliminar la publicidad de un artículo, cambiar el tamaño, tipo y color de la letra, e incluso, el fondo del artículo para hacer una lectura más cómoda a la vista.

Antes del desarrollo de este tipo de aplicaciones, las opciones para la gestión de las páginas web eran o bien el listado de favoritos del navegador o los gestores de marcadores tipo Delicious, el inconveniente de ambos sistemas era que solo almacenaban la dirección url pero no el contenido, pudiendo provocar los llamados enlaces muertos, que provocaban la pérdida de información una vez que la url desaparecía. De esta forma, con las aplicaciones de lectura web estos problemas se han podido solucionar ya que no guardan el link sino todo el texto completo de un periódico, blog o revista. 
Una de las mayores ventajas de estas aplicaciones es que permiten almacenar cualquier página web desde el ordenador, y gracias a la sincronización de la aplicación con su versión para móviles y tabletas se facilita la consulta desde cualquier dispositivo. Asimismo también el etiquetado y clasificación de los artículos favorece la localización posterior. Si no se utilizan este tipo de aplicaciones hay que copiar el contenido desde el navegador y pegarlo en otro documento, sin embargo con éstas, el proceso es más sencillo, solamente es necesario pinchar sobre el icono del plugin del navegador y el proceso se realiza automáticamente.

Estas aplicaciones se pueden utilizar de maneras distintas, desde un único dispositivo, por ejemplo un ordenador de sobremesa, o desde varios, por ejemplo un ordenador, y un smartphone o tableta. En este caso además de instalar en el primer caso el plugin en el navegador del ordenador habrá que instalar la aplicación móvil para el dispositivo correspondiente. La información se vincula a través de la cuenta de usuario creada para tal fin en la página de la aplicación. La mayor parte de este tipo de aplicaciones permite utilizar la cuenta de Facebook, Google+ o Twitter.

\section{Objetivos}

El objetivo de este artículo es analizar, comparar y evaluar las distintas aplicaciones de lectura web. Para ello se comprobarán las aplicaciones, analizando sus ventajas e inconvenientes, su funcionamiento y se compararán.

Para el cumplimiento de este objetivo se realizarán las siguientes acciones:

- Selección de las aplicaciones a evaluar.

- Sistematización de las características.

- Análisis de cada aplicación.

- Comparación.

- Determinación de las mejores aplicaciones que existen de lectura web.

\section{Metodología}

Para el cumplimiento de los objetivos se han estudiado las características de las aplicaciones de lectura web y se han definido una serie de indicadores que son los que permitirán evaluar y comparar las prestaciones de las aplicaciones de lectura web.

Para la localización y selección de las aplicaciones se realizaron búsquedas en Chrome Web Store y en Mozilla Add-ons, que son los portales de aplicaciones, extensiones y temas para los navegadores Google Chrome y Mozilla Firefox. 

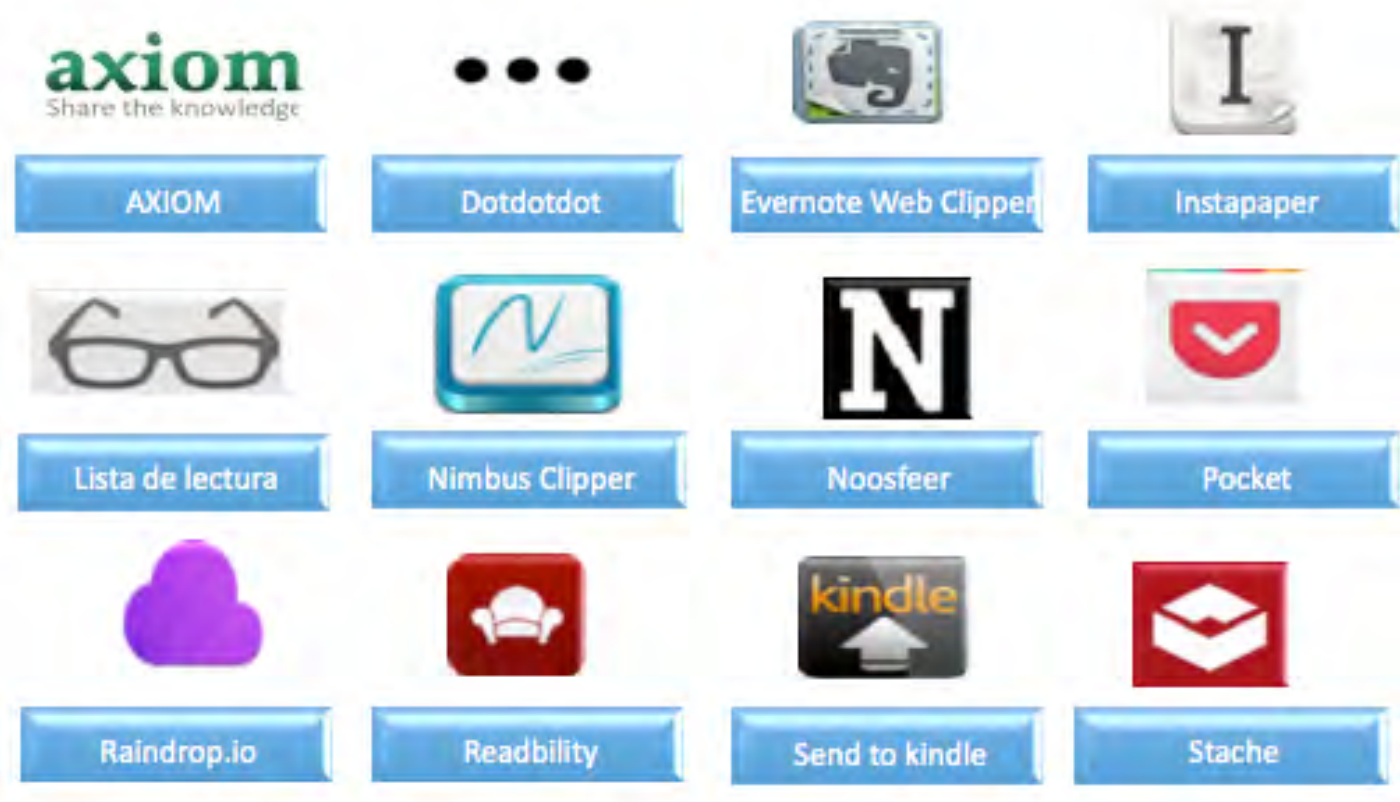

Fig. I Aplicaciones de lectura web

Fuente: elaboración propia

Una vez que se tenían las aplicaciones se fueron definiendo los indicadores que iban a permitir la evaluación. En este sentido y a pesar de que existen trabajos que evalúan otros productos documentales y seleccionan parámetros para evaluar revistas electrónicas (Rodríguez-Gairín, 200I), bases de datos aplicadas al ámbito de enfermería (GuillénRíos, J. F. \& Ayuso-García, M. D., 20I4), la evaluación de bases de datos de Rodríguez Yunta (1998) o las herramientas de evaluación que proponen Martínez Sánchez, Francisco et al. (2002). Hay un trabajo que también selecciona indicadores para la evaluación de aplicaciones de lectura infantil (García-Rodríguez \& Gómez-Díaz, 20I5) pero los parámetros no se ajustan a nuestras necesidades por lo que no se encontró ningún trabajo similar que facilitara la selección de los indicadores. Todos los trabajos citados formulan e implementan indicadores y parámetros para evaluar distintos sitios web, o en el último caso aplicaciones móviles de lectura infantil, sin embargo, estas propuestas no son válidas para la evaluación de aplicaciones web, ya que no se ajustan a las características y opciones que ofrecen. Por lo tanto, la aplicación de indicadores y parámetros de evaluación varían en función de las características de cualquier recurso electrónico, de la tipología y del nivel de profundidad que el evaluador decida emplear (Pinto-Molina, M., 20O4).

Estos trabajos parten de la base del estudio de indicadores y parámetros de evaluación que implementa Codina (2000; 2006). Para la selección de los indicadores y parámetros de las aplicaciones de lectura web, ha servido de guía la metodología utilizada por Codina (2000; 2006) y Pinto Molina (2004). Sin embargo, los parámetros e indicadores que determinan no son válidos para la evaluación de las aplicaciones web, por lo que se han estructurado una serie de parámetros e indicadores propios y específicos que van a determinar la calidad de las aplicaciones presentadas en este estudio. 
En primer lugar se establecieron dos grupos que englobarán los distintos parámetros acorde con su finalidad: Calidad y Prestaciones.

I. Calidad. Según la ISO 9ooo, la calidad es el grado en el que un conjunto de características inherente cumple con los requisitos. En este caso los parámetros agrupados en este grupo indicarán si las aplicaciones satisfacen las expectativas del usuario. Los parámetros que van a evaluar la calidad de las aplicaciones son los siguientes:

I.I. Disponibilidady Accesibilidad: determinará si la aplicación de lectura web es accesible a los usuarios, es decir, si se ha diseñado para que sea compatible con los diferentes navegadores existentes, sistemas operativos móviles, el idioma, etc. Esto conlleva a una mayor visibilidad y por tanto, ofrecer un servicio a mayor número de usuarios.

I.2. Usabilidad: indicará la facilidad o complejidad de uso de la aplicación mediante las características que posee, como por ejemplo, si los iconos son reconocibles e intuitivos al usuario. Se evaluará por tanto, la relación de la aplicación con el usuario, así como el grado de dificultad de interacción con la aplicación.

2. Prestaciones. Son aquellas características y opciones propias de una aplicación web. Las prestaciones ofrecerán una visión global de las opciones y características que poseen las aplicaciones, y por lo tanto, tendrán una importante función, ya que el usuario elegirá la aplicación conforme a sus necesidades y preferencias.

2.I. Personalización: determinará las opciones que ofrece la aplicación para adaptar el texto del artículo, al gusto y necesidades del usuario que pueda tener en un momento determinado. Por ejemplo, si la aplicación ofrece cambiar el color de fondo del artículo, adaptándose al usuario al leer la noticia de día o de noche, ofreciendo los colores de fondo de sepia, negro o blanco.

2.2. Organización de la información: se evaluará cómo organiza la aplicación los documentos que importa el usuario, bien sea a través de carpetas, etiquetas, favoritos o artículos archivados. Al terminar la evaluación de este parámetro, se podrá determinar el rápido acceso a los documentos cuando se quiera disponer de ellos. También se podrá concluir si la aplicación tiene en cuenta al usuario para poder organizar su información, en base de su trabajo, hobbies, estudios o investigaciones.

2.3. Tratamiento de la información: consistirá en comprobar qué opciones tiene por defecto la aplicación en relación al tratamiento de los documentos. Por ejemplo, si da la posibilidad de compartir los documentos con redes sociales o correo electrónico.

A continuación se recogerán los criterios, parámetros e indicadores en una Tabla de manera esquemática. 
Tabla I Criterios, parámetros e indicadores para la evaluación de aplicaciones de lectura web

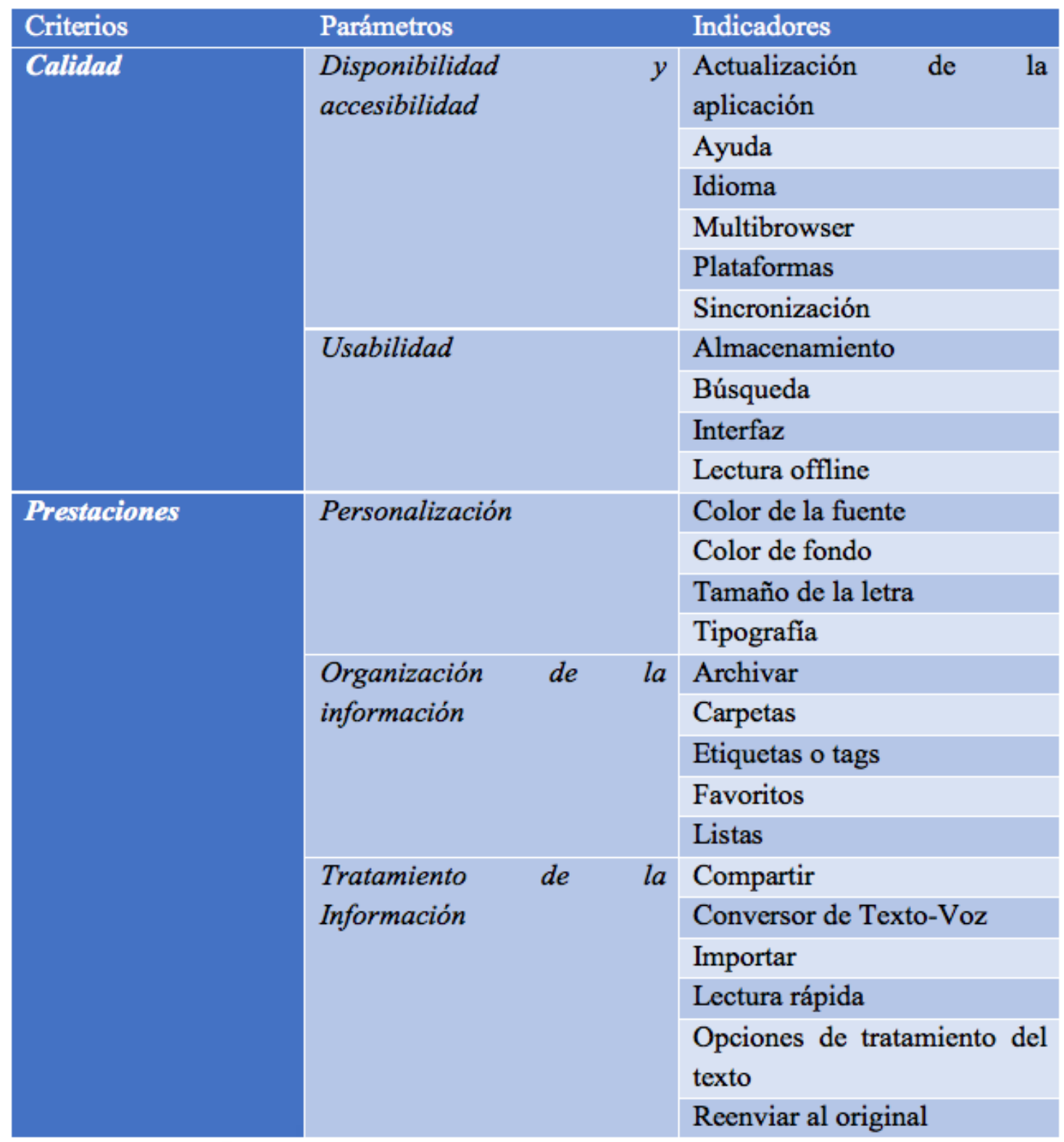

Fuente: Elaboración propia

Una vez definidos los parámetros se fijaron los criterios de evaluación. En este sentido tanto Codina, Ll. (2006) como Ayuso García y Martínez Navarro (2005; 2006) recomiendan puntuar de o a 3 puntos cada característica según su adecuación a la situación que se plantea.

o puntos: se entenderá que ofrece un cumplimiento suficiente, pero con limitaciones.

I punto: cumplimiento adecuado.

2 puntos: un cumplimiento notable.

3 puntos: un cumplimiento excelente. 
En aquellos casos que la respuesta requiera una puntuación de se cumple o no se cumple, se puntuará con i ó o respectivamente. Otros autores Barrera, C.R., NúñezAmaro, S. \& Motola, D. (2006) proponen la puntuación de características binarias como No se cumple $=$ o puntos y Sí se cumple= 3 puntos. Sin embargo, esta evaluación seguirá las pautas descritas por Codina, sí se cumple (I) y no se cumple (o).

\section{Evaluación de las aplicaciones de lectura web}

En la evaluación de las aplicaciones se ha tenido en cuenta las versiones gratuitas. Es importante indicar que el análisis de las mismas se ha hecho a lo largo del mes de mayo de 20I5, por lo que es posible que algunas funcionalidades no aparezcan recogidas en la tabla por ser versiones posteriores. Asimismo, hay que tener en cuenta que las aplicaciones van mejorando cada vez que se actualizan, por lo que están en constante progreso.

La puntuación de las aplicaciones se recogerá en distintas tablas divididas en secciones atendiendo a los parámetros establecidos, que recogerán los indicadores implantados.

Por otro lado, en cada tabla se ha creído oportuno añadir una columna la cual recoja la puntuación obtenida en cada parámetro, cuyo fin será evaluar individualmente cada parámetro, siendo así más sencilla su comparación.

Por último, la puntuación final de todas las aplicaciones de lectura web se fijará en una tabla, que posteriormente será analizada y comentada. 
Tabla 2 Parámetro Disponibilidad y Accesibilidad

\begin{tabular}{|c|c|c|c|c|c|c|c|}
\hline & $\begin{array}{l}\text { Actualización } \\
\text { de la } \\
\text { aplicación }\end{array}$ & Ayuda & Idioma & Multibrowser & Plataformas & Sincronización & $\begin{array}{l}\text { Puntuación } \\
\text { obtenida }\end{array}$ \\
\hline $\begin{array}{l}\text { axiom } \\
\text { Axiom }\end{array}$ & 1 & 0 & 1 & 2 & 0 & 1 & 5 \\
\hline $\begin{array}{l}\bullet \bullet \bullet \\
\text { Dotdotdot }\end{array}$ & 0 & 1 & 0 & 1 & 1 & 1 & 4 \\
\hline $\begin{array}{l}\text { O } \\
\text { Evernote } \\
\text { Web } \\
\text { Clipper }\end{array}$ & 1 & 1 & 3 & 2 & 0 & 1 & 8 \\
\hline$\underbrace{\text { I }}_{\text {paper }}$ Insta & 1 & 1 & 1 & 2 & 3 & 1 & 9 \\
\hline $\begin{array}{r}60 \\
\text { Lista de } \\
\text { Lectura }\end{array}$ & - & 0 & 3 & 0 & 1 & 1 & 5 \\
\hline$\underbrace{}_{\text {bus Clipper }}$ & 1 & 1 & 3 & 1 & 1 & 1 & 8 \\
\hline$\underset{\text { Noosfeer }}{\mathbf{N}}$ & 1 & 1 & 3 & 1 & 2 & 1 & 9 \\
\hline$\underbrace{}_{\text {et }}$ Pock & 1 & 1 & 3 & 2 & 3 & 1 & 11 \\
\hline Raindrop.io & 1 & 1 & 3 & 2 & 2 & 1 & 10 \\
\hline Readability & 0 & 1 & 1 & 2 & 2 & 1 & 7 \\
\hline $\begin{array}{l}\text { Send } \\
\text { to Kindle }\end{array}$ & 1 & 0 & 0 & 2 & 3 & 1 & 7 \\
\hline$\underset{\text { Stache }}{9}$ & 0 & 0 & 0 & 1 & 1 & 1 & 3 \\
\hline
\end{tabular}

Fuente: Elaboración propia

La actualización de la aplicación Lista de Lectura no se ha puntuado debido a que depende del navegador Safari, por lo que se decidió no evaluarla. La ayuda es un parámetro necesario para el usuario, ya que si, tiene alguna duda o consulta de cómo funciona alguna opción pueden acudir a ella y resolver sus dudas, no obstante en algunas aplicaciones de lectura web como Axiom, Lista de Lectura, Send to Kindle y Stache no incorporan esta opción. 
En cuanto al idioma, las aplicaciones Dotdotdot, Send to Kindle y Stache solo tienen la versión en inglés. Por el contrario, las aplicaciones que tienen su versión en cuatro o más idiomas son Evernote Web Clipper, Lista de Lectura, Nimbus Clipper, Noosfeer, Pocket y Raindrop.io. En cuanto a los navegadores soportados, como se ha mencionado anteriormente, Lista de Lectura está disponible solamente para el navegador Safari, en cambio, las aplicaciones de lectura web que soportan entre cuatro y cinco navegadores son Axiom, Evernote Web Clipper, Instapaper, Pocket, Raindrop.io, Readability y Send to Kindle.

En la evaluación de estas aplicaciones, ninguna de ellas se ha considerado multibrowser, es decir, que soporta los seis navegadores más populares existentes (Google Chrome, Internet Explorer, Mozilla Firefox, Safari, Opera y Netscape). Sin embargo, sí hay aplicaciones multiplataforma, es decir, que soportan los sistemas operativos existentes hoy en día (Android, iOS y Windows Phone) como Instapaper, Pocket y Send to Kindle.

Por otro lado, todas las aplicaciones ofrecen la sincronización con distintos dispositivos. Como vemos en esta primera tabla de evaluación, las aplicaciones que tienen una mejor Disponibilidad y Accesibilidad son, en orden de puntuación, Pocket y Raindrop.io. 
Tabla 3 Parámetro usabilidad

\begin{tabular}{|c|c|c|c|c|c|}
\hline & Almacenamiento & Búsqueda & Interfaz & Lectura ofiline & $\begin{array}{l}\text { Puntuación } \\
\text { obtenida }\end{array}$ \\
\hline$\frac{\text { axiom }}{\text { Axiom }}$ & 0 & 1 & 1 & 0 & 2 \\
\hline $\begin{array}{l}\bullet \bullet \bullet \\
\text { Dotdotdot }\end{array}$ & 1 & 2 & 2 & 0 & 5 \\
\hline $\begin{array}{l}\text { Evernote Web } \\
\text { Clipper }\end{array}$ & 0 & 2 & 2 & 0 & 4 \\
\hline I Instapaper & 1 & 0 & 3 & 1 & 5 \\
\hline $\begin{array}{l}60 \\
\text { Lista de Lectura }\end{array}$ & 1 & 0 & 2 & 1 & 4 \\
\hline$\underbrace{}_{\text {Clipper }}$ Nimbus & 0 & 2 & 2 & 0 & 4 \\
\hline $\begin{array}{l}\mathbf{N} \\
\text { Noosfeer }\end{array}$ & 1 & 0 & 2 & 1 & 4 \\
\hline$\checkmark$ Pocket & 1 & 1 & 3 & 1 & 6 \\
\hline Raindrop.io & 1 & 2 & 3 & 1 & 7 \\
\hline Readability & 1 & 1 & 2 & 1 & 5 \\
\hline $\begin{array}{l}\text { Send to } \\
\text { Kindle }\end{array}$ & 1 & 1 & 2 & 1 & 5 \\
\hline Stache & 0 & 2 & 2 & 0 & 4 \\
\hline
\end{tabular}

Fuente: Elaboración propia

El almacenamiento de las aplicaciones Evernote Web Clipper y Nimbus Clipper se ha evaluado como almacenamiento ilimitado en versión de pago (o puntos), ya que las aplicaciones de las que dependen (Evernote y Nimbus Note) ofrecen una versión Premium y Pro en las que añaden más almacenamiento a las aplicaciones que en la versión gratuita. 
Por otro lado, la búsqueda en la aplicación Instapaper, en versión gratuita, no dispone de esta prestación. Solamente ofrece la búsqueda en su versión de pago. Al mismo tiempo, la aplicación Pocket ofrece, en su versión gratuita, una búsqueda sencilla. En cambio, si se quiere disponer de una búsqueda avanzada por palabras clave, etiquetas o anotaciones, se tendrá que adquirir la versión Premium. Las prestaciones que ofrece Pocket en su versión Premium es una biblioteca permanente, realizando una copia de seguridad de todo lo guardado en la aplicación. Una potente búsqueda usando el tema, etiquetas, autor, palabras clave y mucho más. También sugiere unas etiquetas para organizar la lista documentos guardados.

En cuanto a la apariencia visual de la app, las que se han considerado más intuitivas, sencillas de utilizar y comprender son Instapaper, Pocket y Raindrop.io.

Por último, la opción de lectura offline solo la incorporan Instapaper, Lista de Lectura, Noosfeer, Pocket, Raindrop.io, Readability y Send to Kindle. 
Tabla 4 Parámetro personalización

\begin{tabular}{|c|c|c|c|c|c|}
\hline & $\begin{array}{l}\text { Color de la } \\
\text { fuente }\end{array}$ & Color de fondo & $\begin{array}{l}\text { Tamaño de la } \\
\text { fuente }\end{array}$ & Tipografía & $\begin{array}{l}\text { Puntuación } \\
\text { obtenida }\end{array}$ \\
\hline$\frac{\text { axiom }}{\text { Axiom }}$ & 0 & 0 & 1 & 0 & 1 \\
\hline$\bullet$ Dotdotdot & 0 & 1 & 0 & 0 & 1 \\
\hline $\begin{array}{l}\text { Evernote Web } \\
\text { Clipper }\end{array}$ & 1 & 0 & 1 & 1 & 3 \\
\hline I Instapaper & 0 & 1 & 1 & 1 & 3 \\
\hline $\begin{array}{l}60 \\
\text { Lista de Lectura }\end{array}$ & 0 & 0 & 1 & 0 & 1 \\
\hline$\bigoplus_{\text {Clipper }}$ Nimbus & 0 & 0 & 1 & 1 & 2 \\
\hline $\mathbf{N}$ Noosfeer & 0 & 0 & 0 & 0 & 0 \\
\hline$\checkmark$ Pocket & 0 & 1 & 1 & 1 & 3 \\
\hline Raindrop.io & 0 & 1 & 1 & 1 & 3 \\
\hline Readability & 0 & 1 & 1 & 1 & 3 \\
\hline $\begin{array}{ll}\text { Send } \\
\text { Kindle }\end{array}$ & 0 & 1 & 1 & 1 & 3 \\
\hline Stache & 0 & 0 & 0 & 0 & 0 \\
\hline
\end{tabular}

Fuente: Elaboración propia

Como vemos, cambiar el color de la fuente en las aplicaciones de lectura no es una característica que tengan solamente la aplicación de Evernote Web Clipper, o en su caso, Evernote.

Por otro lado, las aplicaciones que no ofrecen ningún parámetro de personalización son Noosfeery Stache. 
Tabla 5 Parámetro Organización de la información

\begin{tabular}{|c|c|c|c|c|c|c|}
\hline & Archivar & Carpetas & $\begin{array}{l}\text { Etiquetas } \\
\text { o tags }\end{array}$ & Favoritos & Listas & $\begin{array}{l}\text { Puntuación } \\
\text { obtenida }\end{array}$ \\
\hline axiom Axiom & 0 & 0 & 1 & 0 & 0 & 1 \\
\hline$\bullet \bullet$ Dotdotdot & 0 & 0 & 1 & 0 & 1 & 2 \\
\hline $\begin{array}{l}\text { Evernote Web } \\
\text { Clipper }\end{array}$ & 0 & 0 & 1 & 0 & 0 & 1 \\
\hline I Instapaper & 1 & 1 & 0 & 1 & 0 & 3 \\
\hline $\begin{array}{l}60 \\
\text { Lista de Lectura }\end{array}$ & 0 & 1 & 0 & 1 & 0 & 2 \\
\hline clipper $\quad$ Nimbus & 0 & 1 & 1 & 0 & 0 & 2 \\
\hline $\mathbf{N}$ Noosfeer & 0 & 0 & 0 & 0 & 0 & 0 \\
\hline$\checkmark$ Pocket & 1 & 0 & 1 & 1 & 0 & 3 \\
\hline Raindrop.io & 0 & 1 & 1 & 0 & 0 & 2 \\
\hline Readability & 1 & 0 & 1 & 1 & 0 & 3 \\
\hline $\begin{array}{ll}\text { Send } \\
\text { Kindle }\end{array}$ & 0 & 0 & 0 & 1 & 0 & 1 \\
\hline Stache & 0 & 0 & 0 & 0 & 0 & 0 \\
\hline
\end{tabular}

La organización de la información puede resultar una prestación de gran importancia para el usuario, ya que podrá administrar y organizar los contenidos en base a sus preferencias, para que sea una tarea más fácil y sencilla recuperar los documentos.

Instapaper, Pocket y Readability ofrecen la opción de archivar los documentos una vez leídos. La opción de crear carpetas para guardar los contenidos se ofrece en Instapaper, Lista de Lectura, Nimbus Clipper y Raindrop.io. 
Añadir etiquetas a los contenidos de las aplicaciones lo ofrecen Axiom. Dotdotdot, Evernote Web Clipper, Nimbus Clipper, Pocket, Raindrop.io y Readability. En cuanto a la opción de favoritos la incluyen Instapaper, Lista de Lectura, Pocket, Readability y Send to Kindle.

Por último, la única aplicación que ofrece crear listas para organizar la información es Dotdotdot.

Las aplicaciones que ofrecen las mejores características en cuanto a la organización de la información son Instapaper, Pocket y Readability.

Tabla 6 Parámetro Tratamiento de la información

\begin{tabular}{|c|c|c|c|c|c|c|c|}
\hline & Compartir & $\begin{array}{l}\text { Conversor } \\
\text { texto-voz }\end{array}$ & Importar & $\begin{array}{l}\text { Lectura } \\
\text { rápida }\end{array}$ & $\begin{array}{l}\text { Opciones } \\
\text { de } \\
\text { tratamiento } \\
\text { del texto }\end{array}$ & $\begin{array}{l}\text { Reenviar } \\
\text { al } \\
\text { original }\end{array}$ & $\begin{array}{l}\text { Puntuación } \\
\text { obtenida }\end{array}$ \\
\hline $\begin{array}{l}\text { axiom } \\
\text { Axiom }\end{array}$ & 1 & 0 & 1 & 0 & 1 & 1 & 4 \\
\hline$\bullet \bullet \bullet \quad$ Dotdotdot & 1 & 0 & 0 & 0 & 1 & 1 & 3 \\
\hline $\begin{array}{l}\text { Evernote Web } \\
\text { Clipper }\end{array}$ & 2 & 0 & 1 & 0 & 1 & 1 & 5 \\
\hline I Instapaper & 1 & 0 & 1 & 1 & 0 & 1 & 4 \\
\hline $\begin{array}{l}60 \\
\text { Lista de Lectura }\end{array}$ & 1 & 0 & 1 & 0 & 0 & 1 & 3 \\
\hline$\underbrace{}_{\text {Nimbus Clipper }}$ & 0 & 0 & 1 & 0 & 1 & 1 & 3 \\
\hline $\mathbf{N}$ Noosfeer & 2 & 0 & 1 & 0 & 0 & 1 & 4 \\
\hline$\checkmark$ Pocket & 2 & 0 & 1 & 0 & 0 & 1 & 4 \\
\hline Raindrop.io & 0 & 0 & 1 & 0 & 0 & 1 & 2 \\
\hline A Readability & 2 & 0 & 1 & 0 & 0 & 1 & 4 \\
\hline $\begin{array}{l}\text { Send to } \\
\text { Kindle }\end{array}$ & 0 & 1 & 1 & 0 & 1 & 1 & 4 \\
\hline Stache & 2 & 0 & 1 & 0 & 0 & 1 & 4 \\
\hline
\end{tabular}


El tratamiento de la información resulta muy diverso entre las distintas aplicaciones de lectura. En primer lugar, las aplicaciones que no ofrecen la posibilidad de compartir los contenidos con otras aplicaciones (redes sociales o correo electrónico) son Nimbus Clipper, Raindrop.io y Send to Kindle. En cambio, las aplicaciones que sí ofrecen esta opción de compartir en tres o cuatro redes sociales son Evernote Web Clipper, Noosfeer, Pocket, Readability y Stache.

La prestación de conversor texto-voz solamente lo ofrece Kindle. Sin embargo, Instapaper en su versión Premium. Asimismo, la lectura rápida solamente la tiene Instapaper.

Las opciones de tratamiento del texto son similares a las que ofrecen algunas aplicaciones de lectura de libros electrónicos, donde puedes realizar notas en el texto, subrayar o destacar palabras o frases. Esta característica la ofrecen las siguiente aplicaciones: Axiom, Dotdotdot, Evernote Web Clipper, Nimbus Clipper y Send to Kindle.

Por último, todas las aplicaciones analizadas ofrecen la opción de volver al documento original.

En las siguientes tablas se muestra una comparación de todos los criterios con la puntuación así como el resumen de todas las puntuaciones. 
Tabla ${ }_{7}$ Comparativa de las aplicaciones de lectura web

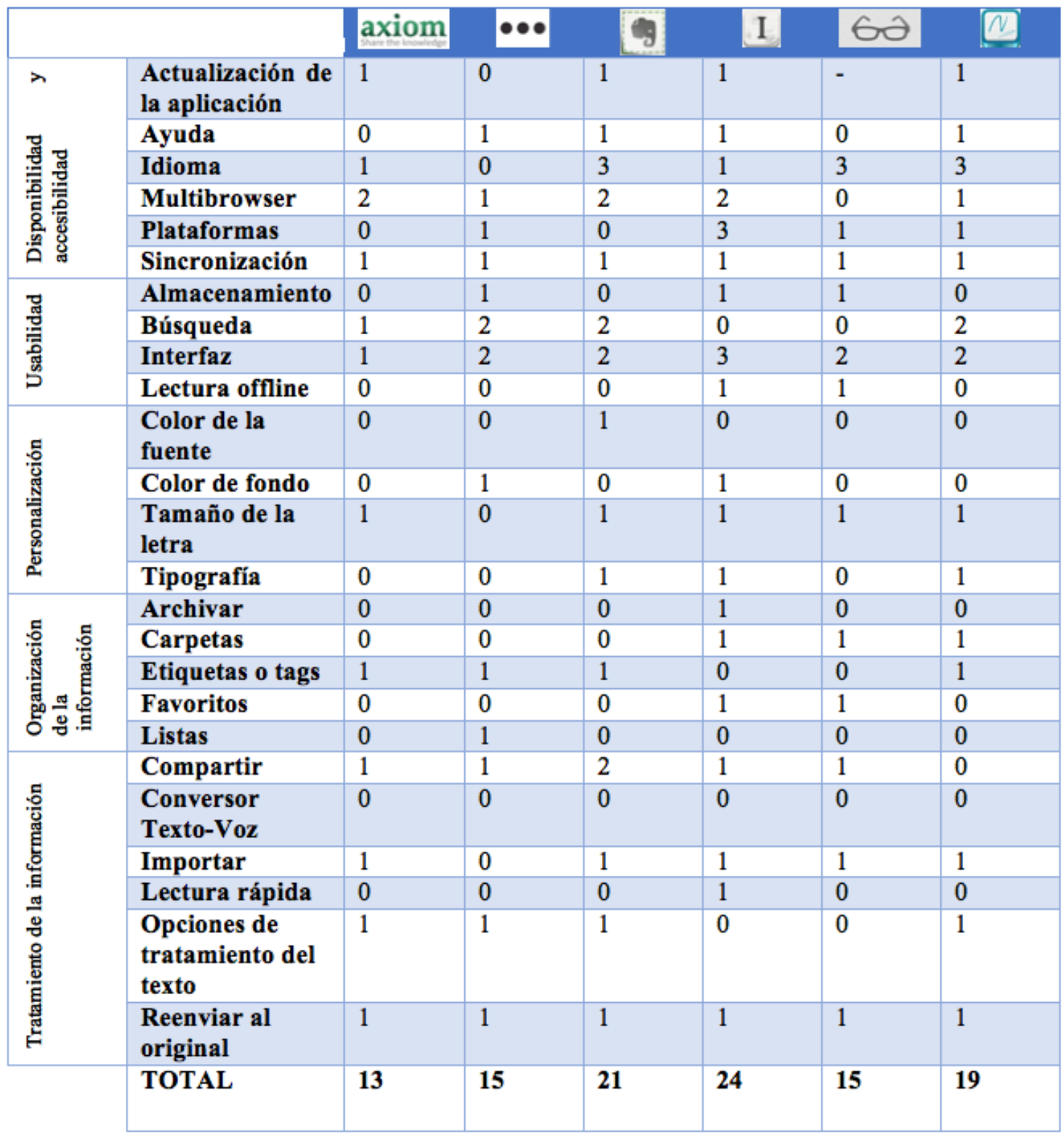


Tabla 7 Comparativa de las aplicaciones de lectura web

\begin{tabular}{|c|c|c|c|c|c|c|c|}
\hline & & & & & & & 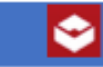 \\
\hline \multirow{6}{*}{ 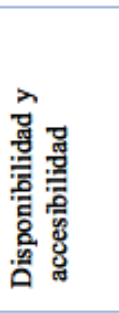 } & $\begin{array}{l}\text { Actualización de } \\
\text { la aplicación }\end{array}$ & 1 & 1 & 1 & 0 & 1 & 0 \\
\hline & Ayuda & 1 & 1 & 1 & 1 & 0 & 0 \\
\hline & Idioma & 3 & 3 & 3 & 1 & 0 & 0 \\
\hline & Multibrowser & 1 & 2 & 2 & 2 & 2 & 1 \\
\hline & Plataformas & 2 & 3 & 2 & 2 & 3 & 1 \\
\hline & Sincronización & 1 & 1 & 1 & 1 & 1 & 1 \\
\hline \multirow{4}{*}{ 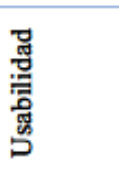 } & Almacenamiento & 1 & 1 & 1 & 1 & 1 & 0 \\
\hline & Búsqueda & 0 & 1 & 2 & 1 & 1 & 2 \\
\hline & Interfaz & 2 & 3 & 3 & 2 & 2 & 2 \\
\hline & Lectura offline & 1 & 1 & 1 & 1 & 1 & 0 \\
\hline \multirow{4}{*}{ 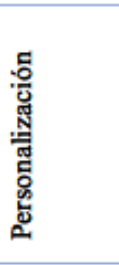 } & $\begin{array}{l}\text { Color de la } \\
\text { fuente }\end{array}$ & 0 & 0 & 0 & 0 & 0 & 0 \\
\hline & Color de fondo & 0 & 1 & 1 & 1 & 1 & 0 \\
\hline & $\begin{array}{l}\text { Tamaño de la } \\
\text { letra }\end{array}$ & 0 & 1 & 1 & 1 & 1 & 0 \\
\hline & Tipografía & 0 & 1 & 1 & 1 & 1 & 0 \\
\hline \multirow{5}{*}{ 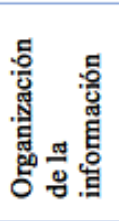 } & Archivar & 0 & 1 & 0 & 1 & 0 & 0 \\
\hline & Carpetas & 0 & 0 & 1 & 0 & 0 & 0 \\
\hline & Etiquetas o tags & 0 & 1 & 1 & 1 & 0 & 0 \\
\hline & Favoritos & 0 & 1 & 0 & 1 & 1 & 0 \\
\hline & Listas & 0 & 0 & 0 & 0 & 0 & 0 \\
\hline \multirow{7}{*}{ 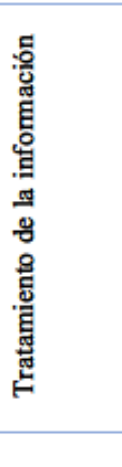 } & Compartir & 2 & 2 & 0 & 2 & 0 & 2 \\
\hline & $\begin{array}{l}\text { Conversor } \\
\text { Texto-Voz }\end{array}$ & 0 & 0 & 0 & 0 & 1 & 0 \\
\hline & Importar & 1 & 1 & 1 & 1 & 1 & 1 \\
\hline & Lectura rápida & 0 & 0 & 0 & 0 & 0 & 0 \\
\hline & $\begin{array}{lr}\text { Opciones } & \text { de } \\
\text { tratamiento } & \text { del } \\
\text { texto } & \end{array}$ & 0 & 0 & 0 & 0 & 1 & 0 \\
\hline & $\begin{array}{l}\text { Reenviar } \\
\text { original }\end{array}$ & 1 & 1 & 1 & 1 & 1 & 1 \\
\hline & TOTAL & 17 & 27 & 24 & 22 & 20 & 11 \\
\hline
\end{tabular}


Tabla 8 Puntuaciones finales

\section{Puntuación Final}

\begin{tabular}{l|l|}
\hline Pocket & 27 \\
Instapaper & 24 \\
Raindrop.io & 24 \\
Readability & 22 \\
Evernote Web Clipper & 21 \\
Send to Kindle & 20 \\
Nimbus Clipper & 19 \\
Noosfeer & 17 \\
Dotdotdot & 15 \\
Lista de lectura & 15 \\
Axiom & 13 \\
Stache & 11 \\
\hline TOTAL & 36 puntos
\end{tabular}

\section{Discusión}

La evaluación de las aplicaciones de lectura web ha concluido en que la mejor aplicación existente hoy en día es Pocket, con una puntuación total de 27 puntos. Seguidamente se encuentran las aplicaciones Instapaper y Raindrop.io, ambas con 24 puntos.

El resultado tan favorable de Pocket es debido a las prestaciones que ofrece en su versión gratuita, son de gran calidad y utilidad. En su versión Premium, solamente ofrece tres características, las cuales a priori no son tan necesarias, como la búsqueda avanzada, biblioteca permanente y etiquetas sugeridas.

Es importante destacar de Pocket que su interfaz es sencilla pero a la vez atractiva, hace que la lectura resulte más cómoda y agradable, gracias a la personalización que ofrece. 
Otras aplicaciones, como por ejemplo Instapaper, en su versión gratuita ofrece unas prestaciones básicas, lo que implica que el usuario pueda decantarse por otras aplicaciones más completas y gratuitas, en lugar de la versión Premium de Instapaper.

Las versiones de pago de Instapaper o Raindrop.io, pueden competir con las prestaciones que ofrece Pocket. No obstante, serán los usuarios los que decidan pagar por una aplicación o bien buscar otras con mejores prestaciones y gratuitas.

Pocket e Instapaper ofrecen una prestación para importar las noticias o tweets que aparecen en Twitter para leerlas posteriormente desde estas aplicaciones. Esta aportación es de gran importancia para los usuarios conectados a esta red social, ya que les permitirá estar informados de todo el contenido que se publica diariamente en Twitter. Actualmente, la mayoría de los usuarios poseen cuentas en redes sociales y están conectados en todo momento, sin embargo, numerosa información, por lo que resulta imposible hacer un seguimiento de todo este contenido. Pero gracias al desarrollo de las aplicaciones de lectura web.

Al mismo tiempo, las aplicaciones que son extensiones de otras apps como Evernote Web Clipper, Send to Kindle y Nimbus Clipper, han obtenido una alta puntuación aun siendo herramientas añadidas a otras aplicaciones.

Por otro lado, no siempre que una aplicación sea de pago, va a tener las mejores prestaciones en relación a aplicaciones de la misma temática. Con esta evaluación se ha podido comprobar que la aplicación de pago, Stache, es la que menos puntuación ha obtenido (II puntos). Esta baja puntuación es debida en gran manera por ser de pago, sin embargo, también porque solamente está diseñada para los sistemas operativos iOS, lo que provoca menor accesibilidad a los usuarios, ya que se encuentra limitada.

Las aplicaciones que cuentan con una baja puntuación en relación a la organización de la información, ya sea debido a que solamente ofrezcan guardar los documentos con etiquetas añadidas o simplemente no ofrezcan ninguna opción, van dirigidas a aquellos usuarios que no necesiten almacenar ni recuperar los documentos ya leídos en la aplicación. Las aplicaciones que han obtenido una puntuación de o-I en este parámetro son Noosfeer, Stache, Axiom y Evernote Web Clipper.

Por último, la puntuación máxima que se ha podido obtener en esta evaluación es de un total de 36 puntos. Aunque Pocket ha obtenido un total de 27 puntos, son 9 puntos de diferencia los que no ha podido conseguir esta aplicación. Esto es debido a que no ofrece las siguientes funcionalidades:

- No es multibrowser. No dispone de una aplicación para el navegador Netscape.

- No ofrece búsqueda avanzada en su versión gratuita. No obstante, sí la posee en la versión Premium.

- No se puede cambiar el color de la fuente.

- No dispone de Conversor texto-voz.

- No proporciona crear carpetas o listas para organizar los documentos. En cambio, sí ofrece las opciones de favoritos, archivar y etiquetas.

- No presenta lectura rápida. 
- No ofrece ninguna opción para anotar, subrayar o comentar los documentos.

- Y por último, solamente se puede compartir en 4 actividades, por lo que ha obtenido 2 puntos. Si facilitara compartir con 5 aplicaciones hubiera obtenido 3 puntos.

\section{Conclusiones}

La evaluación de las aplicaciones de lectura web nos muestran las características y prestaciones que deben de tener para que una aplicación tenga éxito entre los usuarios. Después de su puntuación, Pocket, Raindrop.io, Instapaper y Readability son las mejores aplicaciones existentes actualmente en la web. No obstante, no es casualidad que terceras aplicaciones relacionadas con la prensa, blogs, revistas, etc. den la posibilidad de importar el contenido a estas mismas aplicaciones de lectura web para que las noticias o artículos sean leídos más tarde.

En pocos años hemos visto como prácticas que se habían mantenido casi inmutables a lo largo de los siglos, como es el caso de la lectura, se han ido transformando para dar lugar a nuevas maneras de leer, escribir y editar, una revolución multidimensional que está afectando a las técnicas de producción y reproducción de textos, a los soportes y especialmente a las prácticas de lectura (Furtado, J. A., 2007). El crecimiento de los dispositivos digitales ha revolucionado los hábitos de lectura, cada vez más la lectura se realiza a través de pantallas, bien sean de un ordenador, una tablet o un smartphone. Este crecimiento, es en gran parte, gracias a las ventajas que ofrecen: mayor acceso, disponibilidad y comodidad.

El éxito que han tenido las distintas aplicaciones de lectura, ya sea para libros electrónicos como para otros formatos, es por la personalización del texto al gusto y preferencias del usuario. En un libro impreso podías anotar, subrayar, poner un marcador de páginas, etc. Esa es la esencia que poseen los libros en papel y que tanto gusta a los lectores. Sin embargo, las aplicaciones ofrecen todas estas características y más. Se ha podido comprobar en la evaluación de las aplicaciones, que están adaptadas a los avances tecnológicos y a las preferencias de los usuarios, pudiendo compartir los textos con redes sociales o amigos. La diferencia entre uno y otro reside en el soporte, haciendo que la lectura digital sea más accesible y disponible para el usuario, lo que hace que tenga más auge entre los lectores.

La aceptación de las aplicaciones de lectura web por parte de los usuarios, es debido a su importante utilidad en los tiempos que corren, ya que existe una sobrecarga informativa en la web, que hace imposible leer todo lo que se encuentra navegando por los sitios web. Es por esto, por lo que son necesarias estas aplicaciones, para poder almacenar todos los documentos, artículos, investigaciones, etc. que se encuentren en la web para poder consultarlo más tarde y tener a disposición, de una forma sencilla y organizativa, el contenido cuando se quiera hacer uso de él. 


\section{Referencias bibliográficas}

-Alonso-Arévalo, J., Gómez-Díaz, R., \& Cordón-García, J. A. (2OI5). Ebooks en bibliotecas: gestion, tratamiento y aplicaciones. Buenos Aires: Alfagrama.

- Ayuso-García, M. D. \& Martínez-Navarro, V. (2005). Evaluación de calidad de fuentes y recursos digitales: guía de buenas prácticas. Anales de Documentación, 9, 17-42.

- Ayuso-García, M. D. \& Martínez-Navarro, V. (2006). Metodología de evaluación de recursos en bibliotecas digitales. Parámetros e indicadores de calidad. Ciencias de la Información, 37(I), 25-44. Recuperado de http://www.redalyc.org/articulo.oa?id=I8I4I8O33OO2

- Barrera, C. R., Núñez-Amaro, S. \& Motola-Pedroso, D. (2006). Evaluación de sitios Web en Internet: propuestas para la evaluación de sitios Web de bibliotecas públicas y de salud. Recuperado de http://bvs.sld.cu/revistas/aci/volı4_4_o6/acio4406.htm

- Codina, L. (200o). Evaluación de recursos digitales en línea: conceptos, indicadores y métodos. Revista española de documentación científica, 23(I), 9-44. Recuperado de http:// redc.revistas.csic.es/index.php/redc/article/viewFile/3I5/479

- Codina, L. (2006). Evaluación de calidad en sitios web: metodología de proyectos de análisis sectoriales y de realización de auditorías (Evaluation of quality in web sites: projects methodology for sectorial analyses and audits). Recuperado de http://eprints.rclis. org/8854/

- Cordón García, J. A., Alonso- Arévalo, J., Gómez-Díaz, R. \& Linder, D. (2OI3). Social Reading: Platforms, applications, clouds and tags. Oxford: Chandos.

- Cordón-García, J. A., \& Jarvio-Fernández, A. O. (20I5) • ¿Se está transformando la lectura y la escritura en la era digital? Revista Interamericana de Bibliotecología, 38(2), I37-I45. http://doi.org/ıo.I7533/udea.rib.v38n2ao5

- Encuesta de hábitos y prácticas culturales en España: Septiembre de 2OI5. (2OI5). Madrid: Ministerio de Educación, Cultura y Deporte.

- García Rodríguez, A., y Gómez-Díaz, R. (20I6). Niños y apps: aprendiendo a leer y escribir en digital. Alabe 13. doi: http://dx.doi.org/IO.I564

- García Rodríguez, A., Gómez Díaz, R. (2015). Las demasiadas aplicaciones: parámetros e indicadores para seleccionar las TopAPP de lectura para niños. Anales de Documentación, [S.1.], v. I8, n. 2, oct. 2OI5. ISSN i697-7904. Recuperado de: http://revistas.um.es/analesdoc/article/view/227O 7 I. 
- Gómez-Díaz, R. (2012). La evaluación de las aplicaciones de lectura. Cordón-García, J.A, Carbajo, F., Gómez-Díaz, R., Alonso-Arévalo, J. (Coords). Libros electrónicos y contenidos digitales en la sociedad del conocimiento: mercado, servicios y derechos (pp. 294-295). Madrid: Pirámide.

- Guillen-Ríos, J. F. \& Ayuso-García, M. D. (20I4). Propuesta teórico-metodológica para evaluar la calidad de bases de datos: aplicación al ámbito de enfermería. In Anales de documentación: Revista de biblioteconomía y documentación vol. I7, (2), 5 .

- Hennig, N. (2OI4). Chapter 2: Evaluating Apps. Library Technology Reports, 5 O(8), I5-I7. La lectura en dispositivos móviles: la lectura en E-Reader, Tablet o Smartphone. (2OI4). Madrid: Conecta.

- Maniega, P. L.-N. E. S. D. (2014). App, movilidad de contenidos para la extensión de servicios de información. BiD: Textos Universitaris de Biblioteconomia i Documentació.

- Martínez-Sánchez, F., Prendes-Espinosa, M. P., Alfageme-González, M. B., Amorós-Poveda, L., Rodríguez-Cifuentes, M. T., \& Solano-Fernández, I. M. (2002). Herramienta de evaluación de multimedia didáctico. Recuperado de http://redined.mecd.gob.es/xmlui/ handle/ııт62/95464

- Pinto-Molina, M. (20II).Calidad y evaluación de los contenidos electrónicos. Recuperado de http://www.mariapinto.es/e-coms/eva_con_elec.htm\#e6

- Rodríguez-Gairín, J. M. (200I) Parámetros e indicadores de calidad en la evaluación de una revista electrónica. El caso de $\mathrm{BiD}$ : textos universitaris de biblioteconomia i documentació, en BiD: textos universitaris de biblioteconomia i documentació, 6 .

- Rodríguez-Yunta, L. (1998). Evaluación e indicadores de calidad en bases de datos. Revista Española de Documentación Científica, 2I(I), 9-23.

-Zickuhr, K., \& Rainie, L. (20I4). E-Reading Rises as Device Ownership Jumps: Three in ten adults read an e-book last year; halfown a tablet or e-reader: Pew Research. 